\title{
Mineração
}

\section{O uso da Cokrigagem Co-Locada (collocated cokriging) na integração de dados químicos e mineralógicos no minério de ferro}

\author{
Armando Zaupa Remacre \\ Prof. Dr., Instituto de Geociências - UNICAMP \\ E-mail:armando@ige.unicamp.br \\ Maurílio Camargos Botelho \\ Eng. de Minas, MSc, Gerente de Sistema de Planejamento ao Longo Prazo - Sistema SUL - CVRD \\ E-mail:maurilio@cvrd.com.br
}

\section{Resumo}

Esse artigo apresenta uma técnica de coestimativa Cokrigagem Co-Locada - aplicada à integração de dados mineralógicos e químicos de minério de ferro. Tendo-se como premissa a adoção de um Modelo Markoviano para facilitar a modelagem de covariâncias, o procedimento sugerido consiste na coestimativa da variável mineralógica goethita - GO com o auxílio da variável química Perda por Calcinação - PPC. A utilização dessa técnica é apoiada em: a) Correlação estatística existente entre as variáveis; b) Abundância de amostras da variável PPC, facilitando a modelagem de sua covariância direta. $\mathrm{O}$ método proposto apresenta como principal característica a simplicidade operacional, destacando-se a facilidade do uso da covariância direta da variável Goethita através do uso do Modelo Markoviano. A técnica foi aplicada ao minério de ferro de uma jazida do Quadrilátero Ferrífero e, considerando-se que a proporção de goethita afeta sobremaneira os processos de cominuição, aglomeração e redução, presta-se a auxiliar a caracterização do minério, seja na fase de avaliação de reservas, seja na fase do controle de lavra.

Palavras-chave: krigagem, coestimativa, mineralogia, integração de dados.

\begin{abstract}
This paper presents a coestimation technique Collocated Cokriging - applied for integrating mineralogical and chemical data from iron ore. Adopting, as an assumption, a Markov Model for making the covariances modelling easier, the suggested procedure is made up of coestimating the mineralogical variable Goethite having as auxiliary variable Loss on Ignition. The application of this technique is based on: a) The existing statistical correlation between the two variables; b) Existence of a large amount of Loss on Ignition samples, making its direct covariance modelling easier. The proposed method has, as the most important characteristic, operational simplicity, emphasizing the easily direct covariance modelling of Goethite through the Markov Model 2. The technique was applied to iron ore minerals from a deposit located in the Iron Quadrangle (MG). Considering that the proportion of Goethite affects the comminution, pelletizing and reduction processes, the method is suitable for characterizing the iron ore in both resources modelling and mining control phases.
\end{abstract}

Keywords: kriging, coestimation, mineralogy, data integration, collocated cokriging. 


\section{Introdução}

A indústria de minério de ferro, maior fornecedora de insumos em termos de volumes e custos envolvidos, tem um grande peso na produtividade da indústria siderúrgica, sendo cada vez mais exigida a fornecer minério "sob medida" em termos granulométricos, químicos, mineralógicos e texturais. Assim, esses conhecimentos são de suma importância, haja vista que essas características afetam sobremaneira os processos de aglomeração e redução. A produtividade da sinterização é influenciada diretamente pela porcentagem de minério poroso presente na carga utilizada. As características de resistência mecânica das pelotas são afetadas à medida que varia a quantidade de minério especular na carga de alimentação da pelotização.

Na prática atual da indústria de minério de ferro, a técnica mais tradicional é basicamente qualitativa, não envolvendo modelagem matemática das variáveis envolvidas. Consiste em se fazer a modelagem geológico-tipológica a partir de informações obtidas através de mapeamentos de superfície e resultados de furos de sonda desenhados em seções verticais e horizontais. A partir dessa interpretação, é feito um modelo que serve tanto para estimativa de reservas quanto para controle diário de lavra.

A obtenção de um modelo integrado entre química e mineralogia justificase à medida que pode promover ganhos na melhoria da previsibilidade, e conseqüente produtividade, do minério de ferro nos processos de cominuição, concentração, aglomeração e redução. Libaneo et. al. (2001) destacam a influência da variação da mineralogia no rendimento, desde o pelotamento (aglomeração) até os reatores de redução.

A Figura 1 ilustra a seqüência lógica para a obtenção de um modelo químico mineralógico integrado completo. No primeiro bloco, concentra-se a obtenção dos dados, via coleta de dados espaciais através de furos de sonda e de perfuratrizes e informações geológicas, tais como mapeamento superficial e interpretação de seções verticais e horizontais.
No segundo bloco, concentram-se a parte de tratamento de dados e a análise geoestatística com ênfase para os métodos de coestimativa. No último bloco, de posse das coestimativas químicas e mineralógicas, é possível classificar os blocos de formação ferrífera previamente estimados quimicamente de acordo com os principais tipos mineralógicos.

A aplicação do método proposto (Cokrigagem Co-Locada - CCKO) para as duas variáveis goethita - GO e medida de Perda por Calcinação, PPC, foi definida em função de que (i) estas têm um coeficiente de correlação relativamente elevado, originado pelo fato de que existe uma relação física: a GO é um mineral hidratado e a medida de PPC refere-se justamente à evaporação de moléculas de água que provocam perda de massa de uma determinada amostra de minério, quando esta é submetida a uma temperatura de aproximadamente $1.000^{\circ} \mathrm{C}$. Adicionalmente (ii), segundo Libaneo et. al. (2001), a porcentagem de GO nos minérios tem diversas implicações geosiderúrgicas, destacando-se a importância direta no consumo de energia durante a cominuição, influência na eficácia da flotação e possibilidade de conferir diferentes graus de redutibilidade das pelotas, dependendo de sua proporção.

\section{Metodologia}

A metodologia consistiu em: (i) uma extensa compilação de dados amostrais da área onde, a partir de análises de dispersão, foi definida a priorização das variáveis GO e PPC como as mais viá-

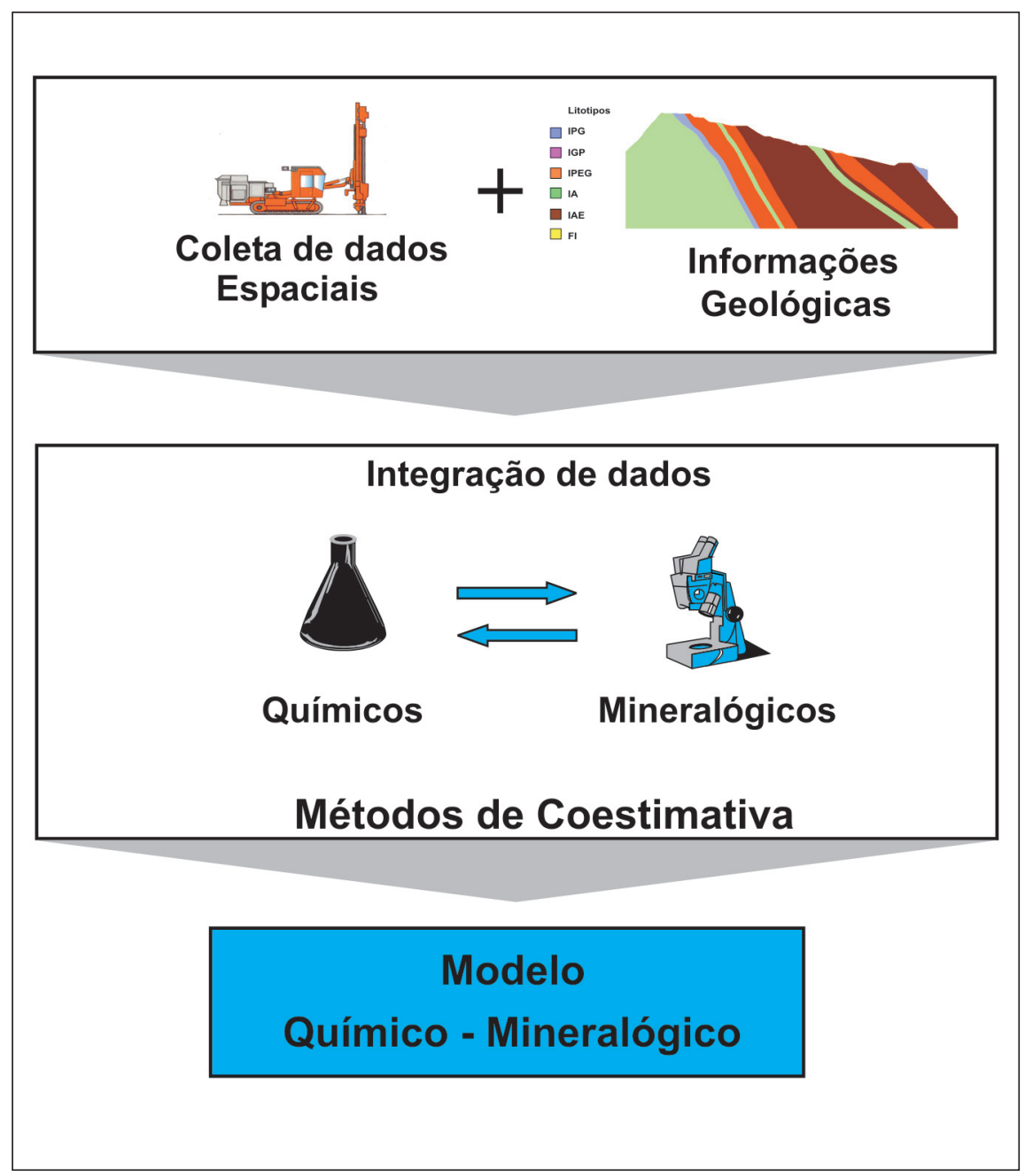

Figura 1 - Seqüência para obtenção de Modelo Químico - Mineralógico. 
veis; (ii) caracterização da variabilidade espacial e a estimativa por Krigagem Ordinária da variável mineralógica $\mathrm{GO}$ e da variável química PPC, haja vista que essas duas estimativas têm funções primordiais na aplicação da CCKO, a saber: a estimativa da GO serve como base de comparação para a eficiência do método proposto; a estimativa do PPC é fundamental para se ter a variável secundária em todos os nós da malha, condição indispensável à aplicação da $\mathrm{CCKO}$; (iii) modelagem da covariância da GO através do uso da hipótese do Modelo Markoviano de Correlação 2 (MM2), haja vista a abundância da variável primária PPC, de mais fácil modelamento direto. Por fim, implementou-se a coestimativa da variável GO, utilizando-se o método CCKO com a consideração da variável química PPC como informação secundária; (iv) Compararam-se os resultados de GO obtidos por meio da CCKO com aqueles obtidos pela $\mathrm{KO}$.

\section{Cokrigagem co- locada (collocated cokriging)}

Com o intuito de solucionar os problemas advindos de malhas densamente amostradas e com dados co-locados, uma solução encontrada por Xu et. al. (apud Wackernagel 1995) consiste na definição de uma estratégia de busca na qual a vizinhança de estimativa da variável secundária é arbitrariamente reduzida a apenas um ponto, ou seja, o ponto mais próximo do ponto $\boldsymbol{u}$ a ser estimado. Dessa maneira, além de se resolverem os problemas de instabilidade, reduz-se a influência dos outros dados secundários não tão bem posicionados em relação ao ponto $\boldsymbol{u}$.

Uma situação hipotética pode ser vista na Figura 2, em que apenas o dado secundário co-locado (verde) é considerado na estimativa. Os outros dados secundários presentes no elipsóide de busca (vermelhos) não entram na resolução do sistema de Cokrigagem Co-Locada.

Essa simplificação da cokrigagem, onde somente um dado secundário co- locado é retido, foi denominada por Xu et al. e Almeida apud Cabral (1998) de Cokrigagem Co-Locada (Collocated Cokriging). O estimador da Cokrigagem Co-Alocada para duas variáveis é uma combinação linear entre os dados da variável primária e único dado secundário. Pode ser escrito como:

$Z_{1}^{*}(u)=\sum_{a 1}^{\mathrm{n} 1(\mathrm{u})} \lambda_{\alpha 1}^{C K O}(u) \cdot Z_{1}\left(u_{a 1}\right)+\lambda_{2}(u) \cdot\left[Z_{2}(u)-m_{2}+m_{1}\right]$

Onde:

$\mathrm{Z}_{1}^{*}(\mathrm{u})=$ estimativa da V.A $\mathrm{Z}_{1}$ no ponto $\boldsymbol{u}(\boldsymbol{x}, \boldsymbol{y}, \boldsymbol{z})$ a ser estimado.

$\mathrm{Z}_{1}(\boldsymbol{u})=$ V.A. primária.

$\mathrm{Z}_{2}(\boldsymbol{u})=$ V.A. secundária,

$\lambda_{\alpha 1}^{\mathrm{CKO}}=$ pesos da variável primária.

$\lambda_{2}=$ pesos da variável secundária.

$\mathrm{n}_{1}(\boldsymbol{u})=$ número de dados (realizações) de $\mathrm{Z}_{1}$ na vizinhança.

$m_{1}=$ média local estacionária da variável primária.

$m_{2}=$ média local estacionária da variável secundária.

A única restrição [esta é a restrição da cokrigagem ordinária "modificada", proposta por Isaaks e Srivastava (1989)] é a de que a soma dos pesos das variáveis primária e secundária deve ser igual a 1 :

$\sum_{a 1}^{\mathrm{n} 1(\mathrm{u})} \lambda_{\alpha 1}(u)+\lambda_{\alpha 2}(u)=1$

Para a obtenção dos modelos de covariância (diretos e cruzados) necessários à resolução do sistema de Cokrigagem Co-Locada, há três possibilidades, de acordo com as hipóteses consideradas:

$1^{\text {a }}$ Possibilidade: construção de um modelo linear de corregionalização (LMC-Linear Model of Coregionalization) Journel (1978), que assume a hipótese de que os modelos criados por combinações lineares são proporcionais a um mesmo modelo básico autorizado.

$2^{\text {a }}$ Possibilidade: construção de um Modelo Markoviano que requer somente a inferência e modelagem da covariância direta da variável primária e o uso do coeficiente de correlação estatística entre as variáveis primária e secundária. Assume a hipótese de que um dado primário co-locado "filtra" a influência de valores da variável secundária posicionados a uma distância $u+h$ do ponto a ser estimado.

$3^{\text {a }}$ Possibilidade: construção de um Modelo Markoviano que requer somente a inferência e modelagem da covariância direta da variável secundária e o uso do coeficiente de correlação estatística entre as variáveis primária e secundária. Assume uma hipótese simétrica àquela da $2^{\mathrm{a}}$ possibilidade. Nesse caso, a premissa é que o dado secundário co-locado "filtra" a influência de outros dados secundários distanciados de $\mathrm{u}+\mathrm{h}$ sobre a variável primária.

\section{Modelo Markoviano 2 - MM2}

Segundo Shmaryan et al. (1998), a variável secundária $z_{2}(\boldsymbol{u})$ é definida em um suporte muito maior que a variável $z_{1}(\boldsymbol{u})$. Em tais casos, a covariância $\mathrm{C}_{2}(\boldsymbol{h})$ é muito mais contínua que $\mathrm{C}_{1}(\boldsymbol{h})$ e a covariância cruzada experimental $\mathrm{C}_{12}(\boldsymbol{h})$ tende a refletir a melhor continuidade de $\mathrm{C}_{2}(\boldsymbol{h})$. Essa constatação fez surgir um modelo alternativo chamado MM2, onde a covariância cruzada $\mathrm{C}_{12}(\boldsymbol{h})$ é feita proporcional à covariância $\mathrm{C}_{2}(\mathrm{~h})$ : 
$C_{12}(h)=\rho_{12}(0) \times C_{2}(h)$

Onde:

$C_{12}(h)=$ Covariância cruzada das variáveis primária e secundária.

$\rho_{12}(0)=$ Coeficiente de correlação estatística entre as variáveis primária e secundária.

$C_{2}(h)=$ Covariância direta da variável secundária.

A hipótese no MM2 é de que o dado secundário $z_{2}(\boldsymbol{u})$ co-locado filtra a influência, na estimativa de $Z_{l}(\boldsymbol{h})$, de quaisquer outros dados secundários posiocionados em $\boldsymbol{u}+\boldsymbol{h}$. A Figura 3 mostra a configuração de filtragem do MM2.

\section{Localização geral da área em estudo e geologia}

A área em estudo é denominada Alegria 1/6, de propriedade da Samarco Mineração S/A, e encontra-se inserida no Complexo Alegria. Segundo Kaneko (1999), "a jazida de ferro de Alegria $1 / 6$ faz parte da estrutura tectônica Sinclinal de Alegria, que possui um eixo de direção NW-SE, caindo para SE, com a aba sul tendo direção N-S e a aba norte, direção E-W. É constituída por Itabiritos pertencentes à Formação Cauê, Grupo Itabira, Supergrupo Minas, incluindo também corpos hematíticos, rolado, canga e algum solo laterítico".

Em um trabalho enfatizando a definição de tipos de itabiritos para alimentação da concentração mineral na Samarco, Hasui et. al. apud Kaneko (1999) citam que os itabiritos da jazida em estudo têm, de acordo com a composição mineralógica do mineral-minério predominante, cinco tipos distintos, sendo eles: itabirito anfibolítico, itabirito martítico, itabirito goethítico, itabirito especularítico, itabirito magnetítico.

Em virtude do enfoque desse trabalho, que é a estimativa e a co-estimativa de dados mineralógicos dentro do corpo de Itabirito, não serão adotadas as classificações tipológicas identificadas por Kaneko (1999), mas somente a classificação litológica. Por-
Alegria 1/6 é extensamente amostrada por furos de perfuratriz, que têm o objetivo inicial de preparar furos para a colocação de explosivos para detonação do minério. A concepção é a de amostrar, através da coleta do pó gerado durante a furação, o minério e analisá-lo quimicamente. Dessa forma, é possível a obtenção de um grande número de resultados químicos a um custo muito inferior ao custo de furos de sonda rotativa (que são utilizados para testemunhagem).

\section{Análise estatística dos dados mineralógicos}

Foi feita uma análise estatística com o intuito de se ter referência acerca dos parâmetros básicos que regem a distribuição das porcentagens de GO medidas nos furos de sonda. Também serviu

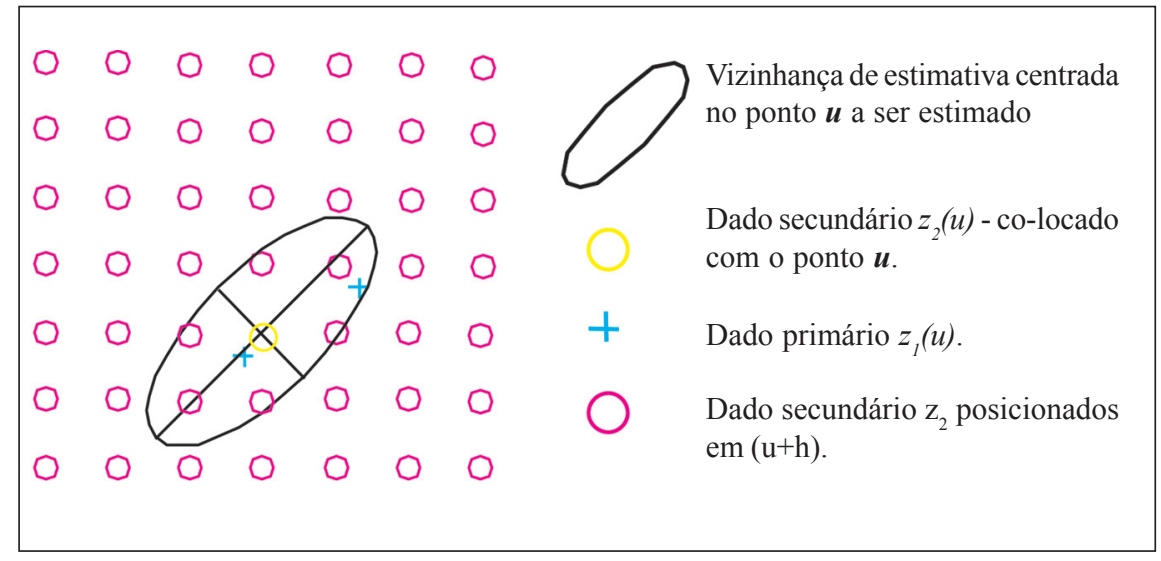

Figura 2 - Estratégia de busca reduzida ao dado secundário co-locado.

\section{Modelo Markoviano 2 - MM2}

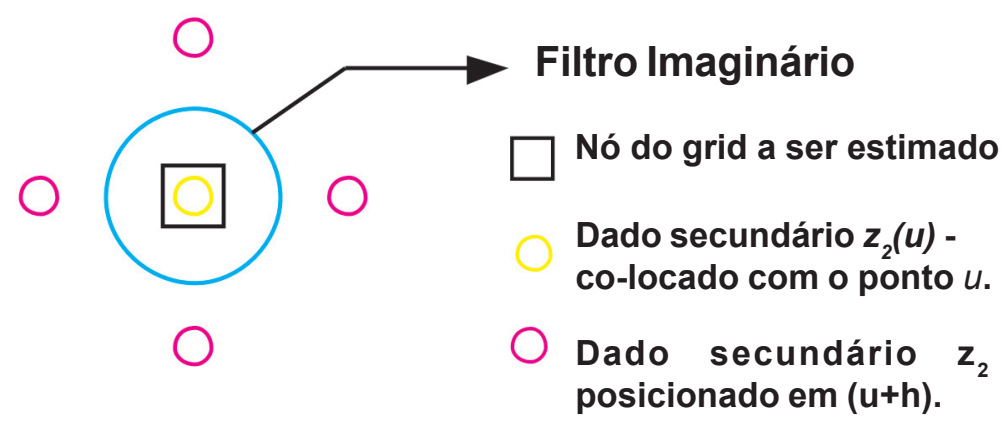

Figura 3 - Ilustração da hipótese de filtragem considerada no Modelo Markoviano 2. 


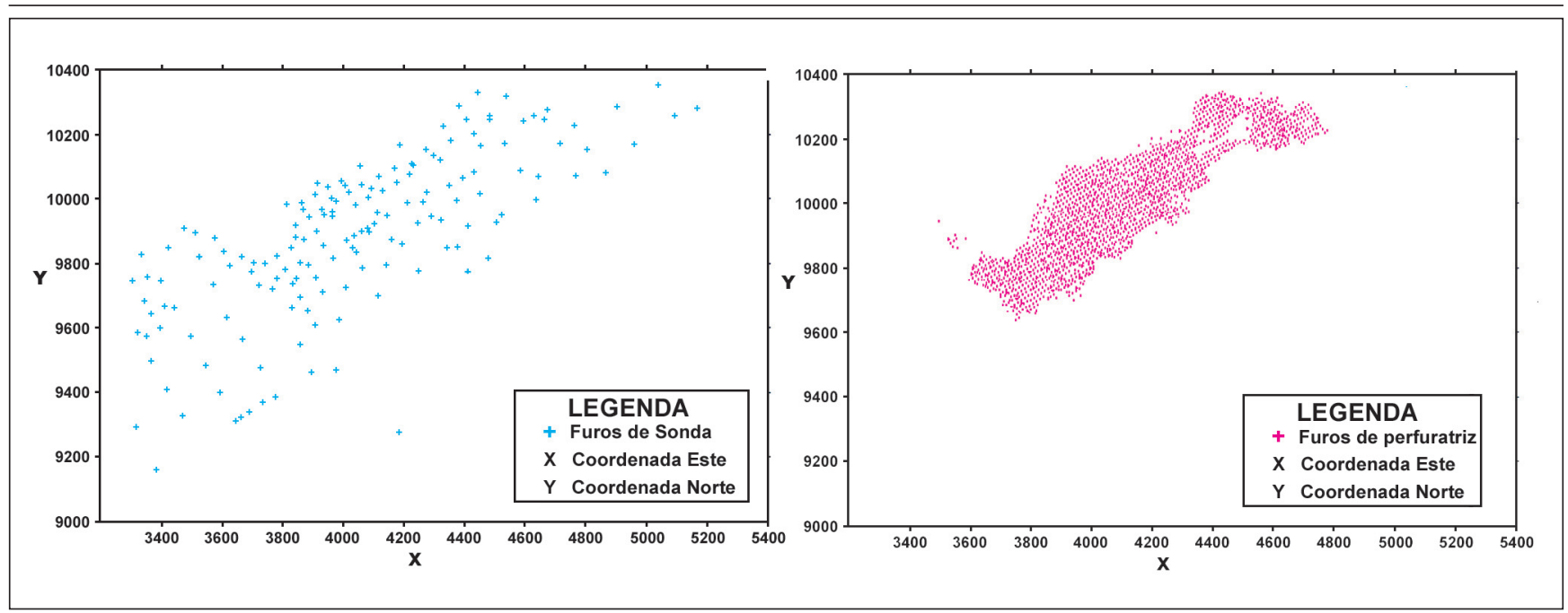

Figura 4 - Mapa-base dos furos de sonda e furos de perfuratriz.

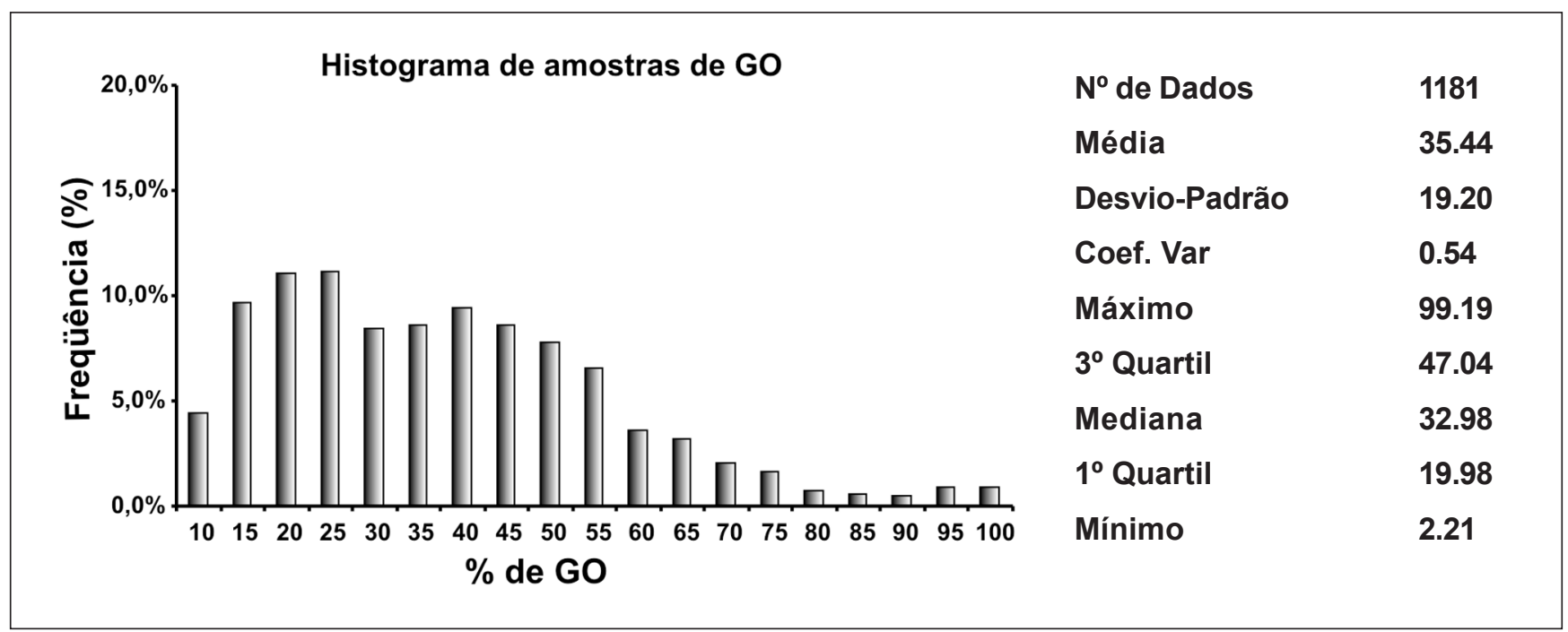

Figura 5 - Histograma e resumo estatístico da \% de GO.

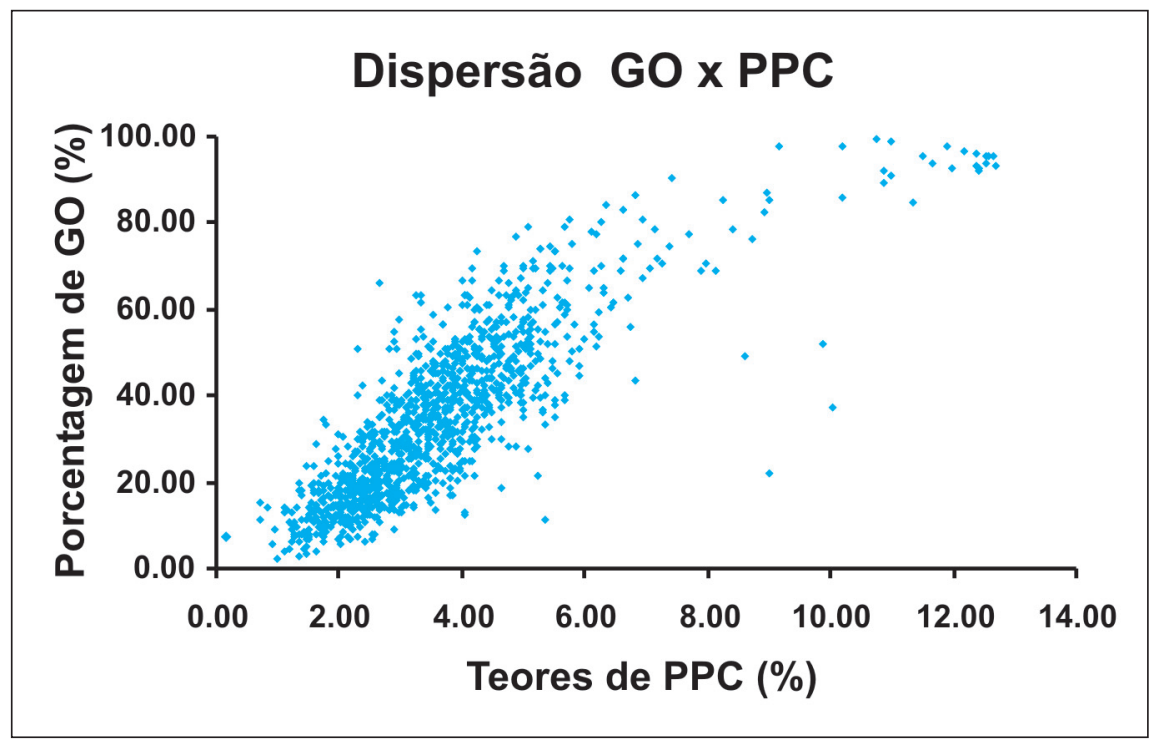

Figura 6 - Diagrama de dispersão GO - PPC. como forma de se verificar a dispersão com a variável química PPC. As Figuras 5 e 6 mostram, repectivamente, a forma de distribuição das porcentagens de $\mathrm{GO}$ e o diagrama de dispersão GO - PPC.

A Krigagem Ordinária da variável química PPC servirá para a constituição do Background ou, em outros termos, para se terem os valores da variável secundária (PPC) co-locados em todos os nós do malha em que se deseja proceder a Cokrigagem Co-Locada da variável mineralógica GO. Foi feita uma análise estatística com o objetivo de se ter referência a respeito dos parâmetros básicos que regem a distribuição dos teores químicos. A Figura 7 mostra o histograma dos teores de PPC obtidos através das 


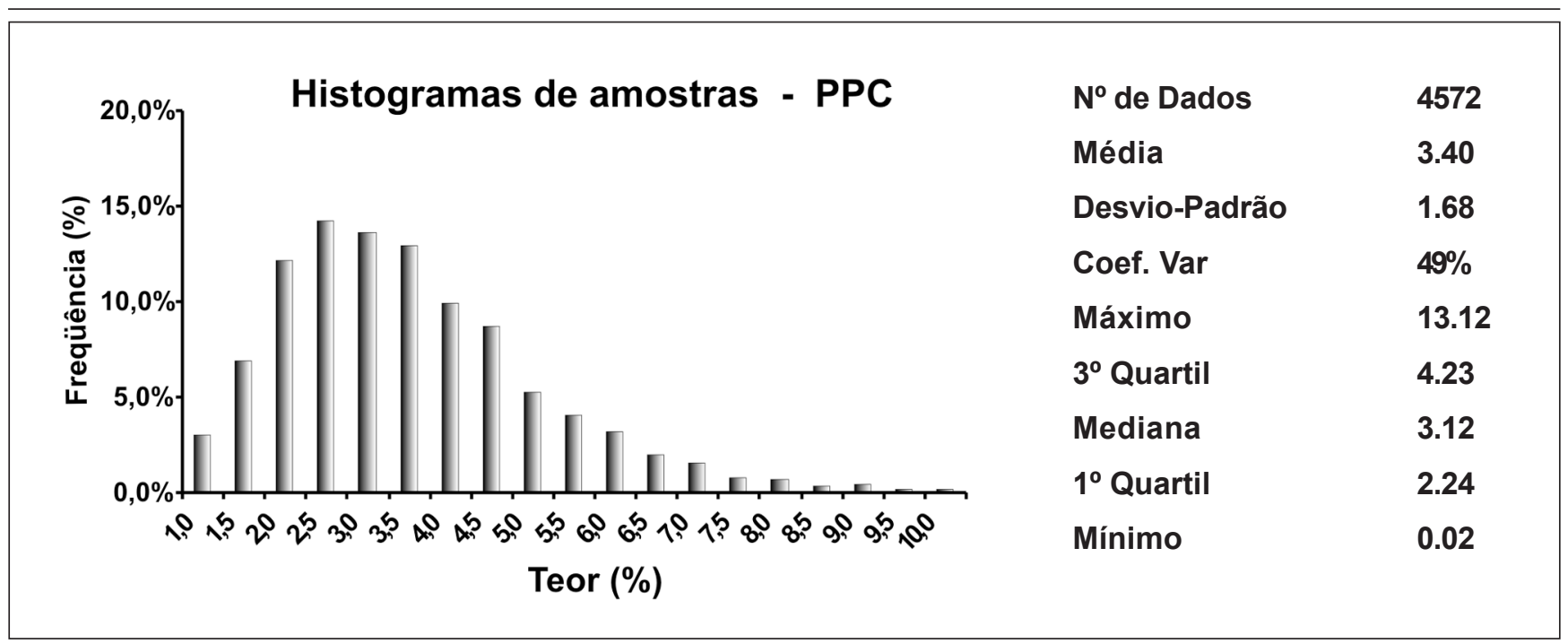

Figura 7 - Histograma e resumo estatístico dos teores de PPC.

amostras dos furos de perfuratriz e de sonda.

Os resultados da Krigagem Ordinária da variável química PPC são imprescindíveis para a implementação da Cokrigagem Co-Locada da variável GO. A Tabela 1 e a Figura 8 trazem, respectivamente, um resumo estatístico dos valores krigados e um histograma dos teores krigados.

\section{Adaptação da Cokrigagem Co-Locada original}

O uso da Cokrigagem Co-Locada na área em questão deve sofrer adaptações devido à configuração dos dados amostrais. Nesse caso, tem-se uma situação de heterotopia parcial, ou seja, existem amostras de mineralogia com as mesmas coordenadas $\boldsymbol{u}(\boldsymbol{x}, \boldsymbol{y}, \boldsymbol{z})$ de amostras de química, mas também existem amostras químicas em locais que não se têm mineralogia.

Diante do exposto, a prática da Cokrigagem Co-Locada na área em questão irá requerer que se estime o valor da variável secundária PPC em todos os nós do malha. Esse procedimento foi feito através da Krigagem ordinária. Somente assim o "background", que é a variável química PPC, estará co-locado nos nós
Tabela 1 - Resumo estatístico dos resultados da Krigagem Ordinária do PPC.

\begin{tabular}{l|c|c}
\hline \multicolumn{1}{c|}{ Variável: PPC } & Valores Krigados & Variância \\
\hline No de Dados $^{\text {Média }}$ & 4129 & 4129 \\
\hline Desvio-Padrão & 3.53 & 1.07 \\
\hline Coeficiente de Variação & 1.31 & 0.37 \\
\hline Máximo & $37.11 \%$ & 34.34 \\
\hline $3^{\circ}$ Quartil & 11.01 & 2.13 \\
\hline Mediana & 4.11 & 1.32 \\
\hline $1^{\circ}$ Quartil & 3.28 & 1.07 \\
\hline Mínimo & 2.65 & 0.78 \\
\hline
\end{tabular}

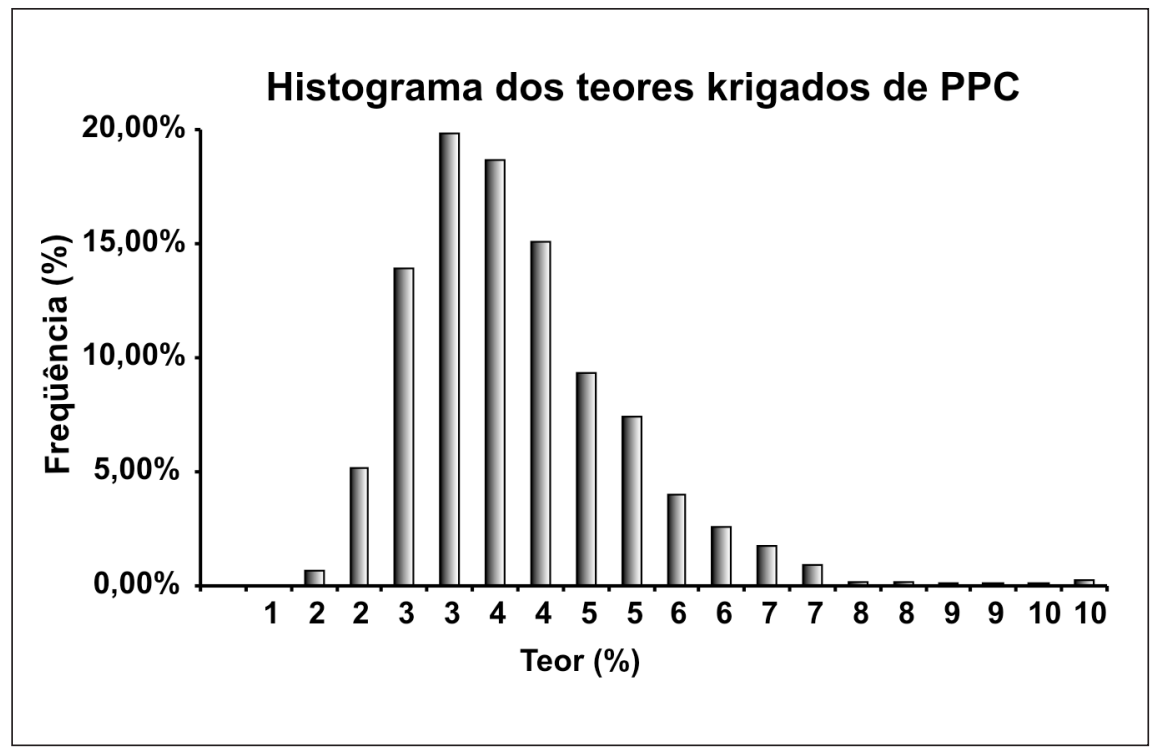

Figura 8 - Histograma dos teores krigados de PPC. 
Armando Zaupa Remacre et al.

do malha onde se deseja fazer a co-estimativa da variável mineralógica GO, como mostra a Figura 9.

Por outro lado, não será necessário, como sugerido no roteiro de Journel (1998), estimar as variáveis secundárias nos pontos onde se têm as variáveis primárias. Como, nos furos de sonda, há um número razoável de locais onde se têm a química e a mineralogia analisadas (situação de isotopia), o coeficiente de correlação para $\mathrm{h}=0$ pode ser calculado diretamente.

Segundo Journel (1998), para uma situação tradicional de aplicação da Cokrigagem Co-Locada, há a necessidade de se estimarem os valores da variável secundária nos pontos onde se tem amostras da variável primária, com o intuito de se calcular o coeficiente de correlação para $\mathrm{h}=0$. Esse procedimento se faz necessário em casos de heterotopia total, onde não existem amostras das variáveis primárias e secundárias nas mesmas posições espaciais.

No estudo de caso, ora apresentado, existem várias amostras da variável primária $(\mathrm{GO})$, coletadas nas mesmas posições espaciais da variável secundária (PPC), permitindo o cálculo direto do coeficiente de correlação para $h=0$, que é de 0,83 .

\section{Comparação por parâmetros estatísticos}

Os resultados da Krigagem Ordinária $(\mathrm{KO})$ e da Cokrigagem Co-Locada $(\mathrm{CCKO})$ da variável mineralógica $\mathrm{GO}$ foram sumarizados na Tabela 2 de forma a possibilitar a comparação sob o ponto de vista estatístico. Pode-se notar que, em termos médios, os dois resultados são bastante semelhantes, sendo que a diferença das médias é de apenas 1.2\%. A KO produziu valores mais atenuados, haja vista que o seu coeficiente de variação é cerca de 3 pontos percentuais menor que o da CCKO. Por último, verifica-se que a variância do erro de estimativa na CCKO é menor que na $\mathrm{KO}$, sendo sua diferença de $29 \%$.
Em continuação à comparação estatística, procedeu-se a análise de dispersão dos teores de GO (co)estimados pelos dois métodos, $\mathrm{KO}$ e CCKO, mostrando se, estatisticamente, a distribuição dos teores são semelhantes ou não. Com o objetivo de proporcionar uma análise diferenciada para grupos que têm densidades amostrais diferentes, evidenciada por menores variâncias de estimativa, foram selecionados dois grupos, segundo a distribuição acumulada das variâncias de estimativa dos blocos estimados por KO, que é o método referência. A partir dessa seleção foram gerados dois grupos, cujas características estatísticas podem ser assim definidas:

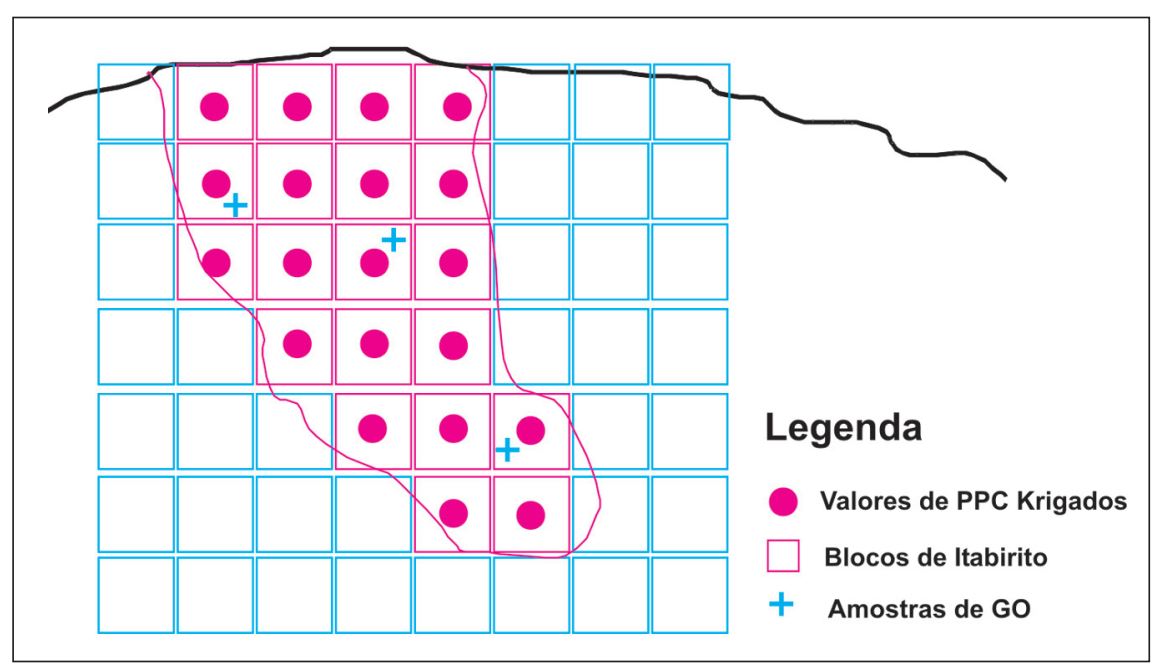

Figura 9 - Seção vertical ilustrando a variável PPC estimada por KO.

Tabela 2- Resumo estatístico das estimativas de GO por KO e CCKO.

\begin{tabular}{l|c|c|c|c}
\hline \multicolumn{1}{c|}{ Variável: GO } & KO & Variância & CCKO & Variância \\
\hline $\mathrm{N}^{\circ}$ de Dados & 4129 & 4129 & 4129 & 4129 \\
\hline Média & 33.18 & 12.73 & 33.59 & 9.02 \\
\hline Desvio-Padrão & 14.22 & 4.12 & 15.70 & 2.60 \\
\hline Coeficiente de Variação & $43.54 \%$ & $32.35 \%$ & $46.75 \%$ & $28.86 \%$ \\
\hline Máximo & 98.28 & 21.36 & 97.53 & 23.55 \\
\hline $3^{\circ}$ Quartil & 41.42 & 15.89 & 43.78 & 10.67 \\
\hline Mediana & 31.41 & 31.01 & 32.14 & 8.79 \\
\hline $1^{\circ}$ Quartil & 21.48 & 9.39 & 21.32 & 7.06 \\
\hline Mínimo & 2.16 & 3.48 & 0.35 & 3.88 \\
\hline
\end{tabular}


Esse fato indica que, em situações mais favoráveis de configuração de dados amostrais (locais onde se tem uma menor variância do erro de estimativa), a Cokrigagem Co-Locada fornece resultados bastante próximos da $\mathrm{KO}$, que é um método consagrado para variáveis regionalizadas.

\section{Funções de recuperação}

As funções de recuperação ou curvas de parametrização fornecem as tonelagens para cada teor de corte. Nesse trabalho serão usadas para medir a eficiência de métodos de estimativa quanto às perdas por seleção errônea de blocos de lavra, seja na fase de classificação de reservas ou otimização de cava final, seja na etapa de controle diário de lavra. Essas perdas podem ser quantificadas em termos de metal contido, de benefício econômico e de tonelagem de minério (ver Cruz Jr., 1998 e Remacre \& Cornetti, 1999).

Nesse estudo de caso, diante do fato de não se terem os valores reais de GO nos blocos, foram construídas curvas de tonelagem teor para os resultados da estimativa por CCKO da variável GO, levando-se em consideração os resultados estimados pela $\mathrm{KO}$ como reserva ideal, até porque a $\mathrm{KO}$ ainda é um método-padrão na indústria mineira como estimativa. Dessa forma, os resultados da CCKO são comparados relativamente aos resultados da $\mathrm{KO}$.

Em termos de recuperação de metal, os resultados obtidos na CCKO para o Grupo 1 mostram que a diferença de metal contido em relação à reserva ideal, estimada por KO, é muito semelhante, de cerca de $1 \%$, situando-se na faixa de erro da KO. Nas curvas de proporção de tonelagem, para a mesma faixa de teores de corte, as diferenças também são pequenas, inferiores a 3\% (Figura 12).

Também no Grupo 2, onde há uma menor densidade amostral de GO para utilização nas (co)-estimativas, as diferenças entre os dois métodos mostraram-se pequenas. Tanto na recuperação de metal contido - Q, quanto na proporção de Tonelagem - T, as diferenças oca-

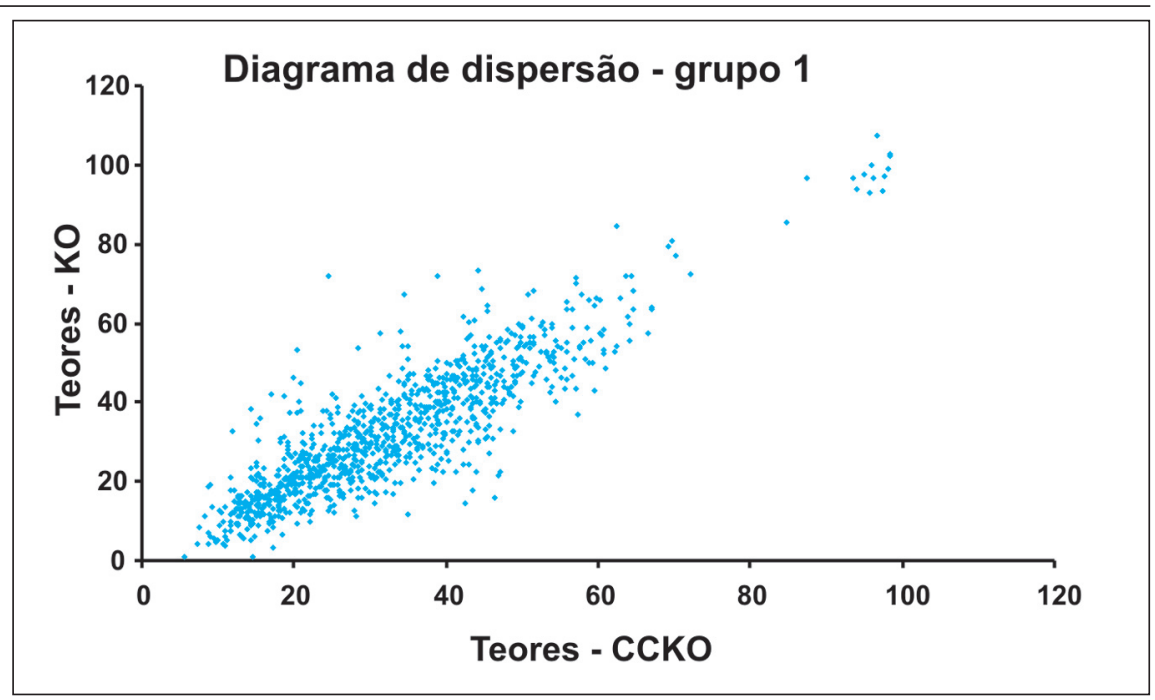

Figura 10 - Diagrama de dispersão de teores de GO do Grupo 1.

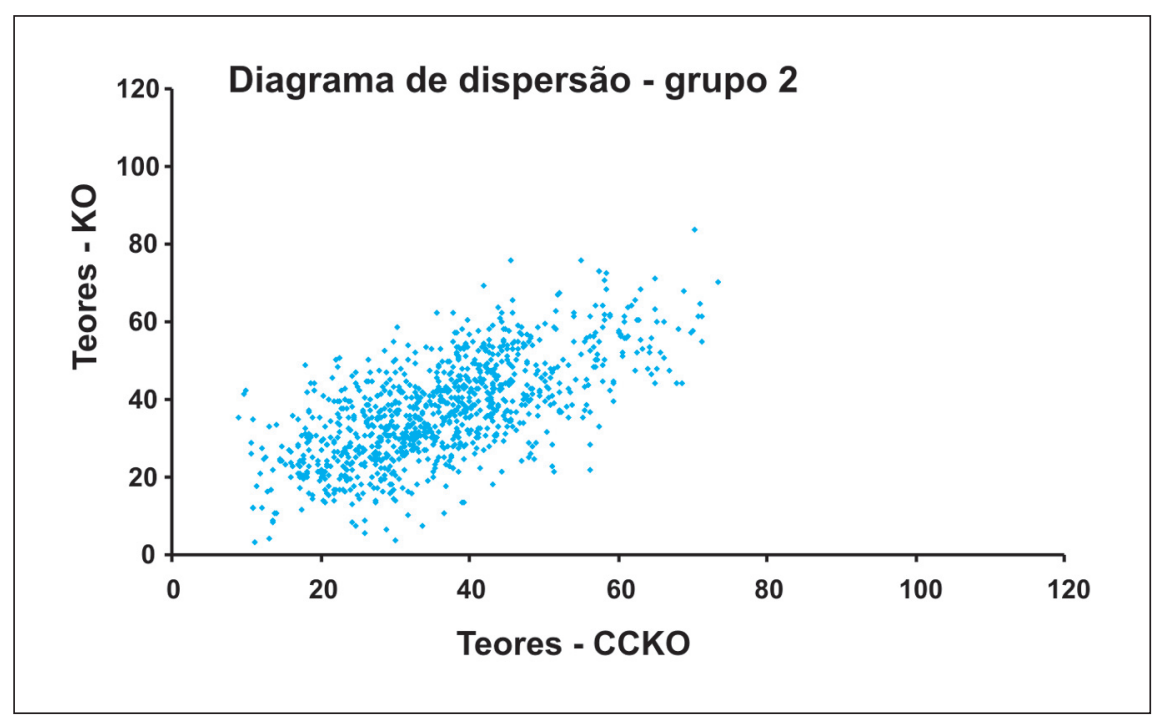

Figura 11 - Diagrama de dispersão de teores de GO do Grupo 2.

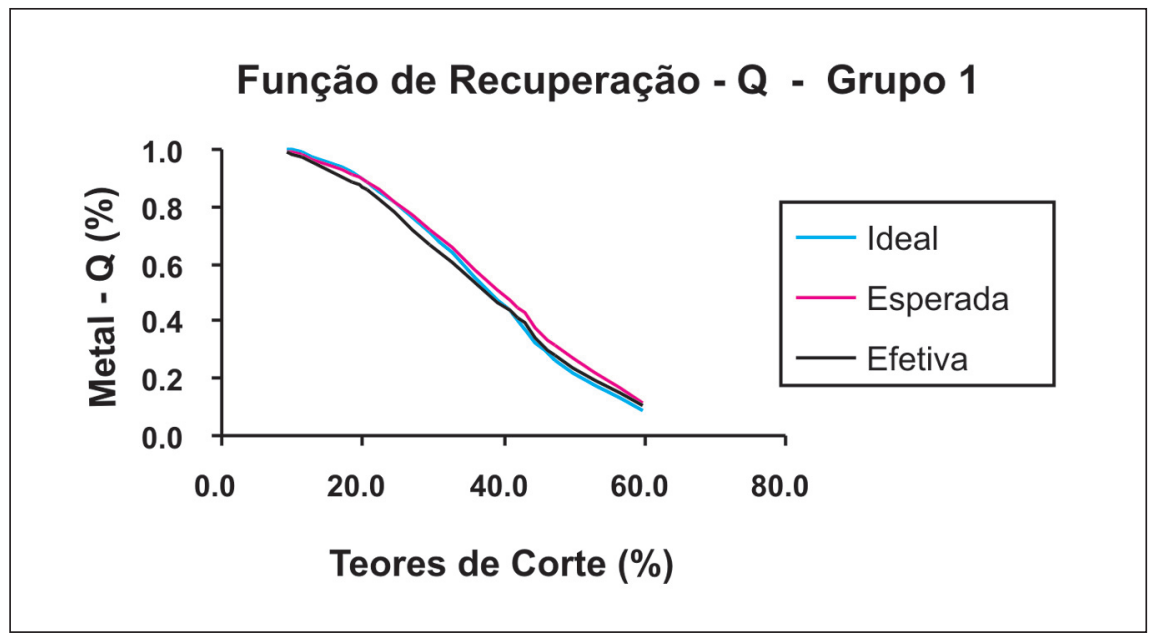

Figura 12 -Metal contido (Q) em reserva classificada com base na CCKO - Grupo 1. 
sionadas pelo uso da CCKO, em relação à reserva ideal (obtida por KO), foram inferiores a $4.9 \%$. Esse resultado mostra que, mesmo em condições de amostragem mais escassa, o uso da CCKO é viável, não gerando diferenças significativas, se comparado à utilização da KO (Figura 13).

\section{Conclusões}

As comparações mostraram que, em termos gerais, os resultados da Cokrigagem Co-Locada - CCKO são estatisticamente equivalentes em relação à Krigagem Ordinária - KO. Portanto o método (CCKO) pode ser aplicado em uma situação de integração parcial de dados químicos e mineralógicos em minério de ferro, prestando-se a constituir um modelo estimado para fins de avaliação de reservas, otimização de cavas finais e controle de lavra. Em termos específicos, há que se destacarem as seguintes conclusões sobre a implementação da Cokrigagem Co-Locada - CCKO em minério de ferro:

1) Uma das restrições à aplicação da CCKO é o fato de ser necessário que a variável secundária esteja presente em todos os nós da malha. A adaptação proposta, em que a variável secundária PPC é estimada por Krigagem Ordinária, feita para possibilitar a implementação da CCKO da variável mineralógica GO, mostrou-se viável, podendo ser aplicada em outros casos.

2) A aplicação da CCKO em integração de dados mineralógicos é especialmente indicada nos casos em que a variável primária for escassamente amostrada, com problemas de ajuste em seu variograma. Nesses casos, como é mostrado nesse trabalho, a escolha do MM2 facilita o ajuste da covariância primária, que é feita através do modelo da covariância secundária e do coeficiente de correlação.

3) A seleção de reservas feita através de um modelo estimado por CCKO, como mostrado no item anterior, apresenta pequenas diferenças, se comparada à seleção baseada em um modelo estimado por KO. Esse fato foi evidenciado, tanto na situação de baixas variân-

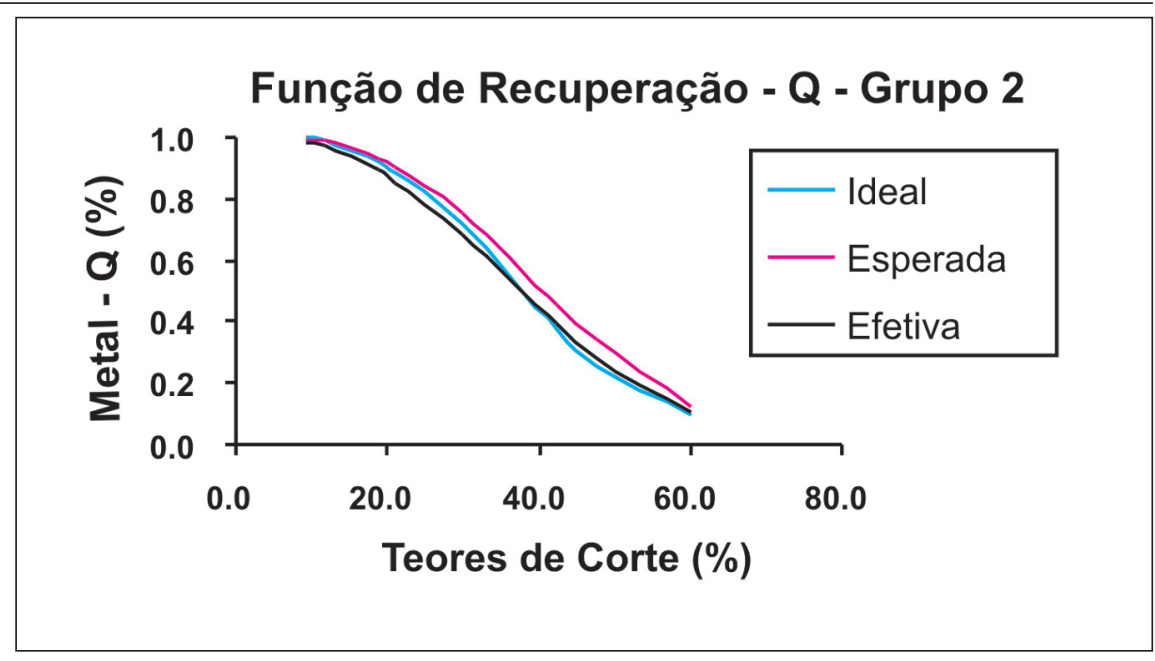

Figura 13 - Metal contido (Q) em reserva classificada com base na CCKO - Grupo 2.

cias de erro de estimativa (Grupo 1), onde as perdas de metal contido não foram superiores a $1 \%$, quanto na situação de maiores variâncias de erro de estimativa, ocasionada por uma menor densidade amostral de GO (Grupo 2).

Assim, conclui-se que o uso da CCKO é recomendável nas situações em que se tiver uma baixa densidade amostral, haja vista que seus resultados mostraram-se semelhantes à KO. Atente-se para o fato de que é possível estimar a GO com a ajuda do PPC, onde antes só era possível krigar a GO com poucas amostras.

\section{Agradecimentos}

Os autores agradecem à CVRD e à SAMARCO MINERAÇÃO. Em especial ao Eng. Frederico A. R. do Carmo (CVRD) pela colaboração na fase de montagem desse artigo. Agradecem igualmente aos dois revisores.

\section{Referências bibliográficas}

BOTELHO, M. С. O uso de cokrigagem co-locada ("collocated cokriging") na integração de dados químicos e mineralógicos no minério de ferro. Campinas: Instituto de Geociências, Departamento de Geologia e Recursos Minerais - UNICAMP. 2001. (Dissertação de Mestrado).

CABRAL. Comportamento de ponderadores e influência de fatores operacionais $e$ estruturais em processos de estimação por diferentes formas de cokrigagem. Belo
Horizonte: Universidade Federal de Minas Gerais, Curso de Pós Graduação em Engenharia Metalúrgica e de Minas, 1998. (Proposta de Tese).

CRUZ JUNIOR, A. C. Análise das funções de recuperação para a parametrização de reservas minerais. Campinas: Instituto de Geociências, Departamento de Geologia e Recursos Minerais - UNICAMP. 1998. (Dissertação de Mestrado).

KANEKO, K. M. Caracterização tipológicamineralógica, estrutural e modelagem geométrica tridimensional da jazida de Ferro de Alegria 1 e 6 - Quadrilátero Ferrífero. Rio Claro: Instituto de Geociências e Ciências Exatas - UNESP. 1999. (Dissertação de Mestrado).

ISAAKS, E., SRIVASTAVA, R. An introduction to applied geostatistics. Oxford University Press Inc. New York: 1989.

JOURNEL, A., HUIJBREGTS, C. J. Mining geostatistics. New York: Academic Press, 1978.

JOURNEL, A. Markov models for cross covariances. Stanford Center for reservoir forecasting - SCRF. Stanford: Stanford University, 1998. v.2.

LIBANEO, C. A. F. et al. Classificação mineralógica, textural e granulométrica de detalhe de minério de ferro ("pellet feed") e suas implicações geosiderúrgicas. In: SIMPÓSIO BRASILEIRO DE MINÉRIO DE FERRO, 3. Ouro Preto: Universidade Federal de Ouro Preto - UFOP, 2001.

REMACRE, A. Z., CORNETTI, M. A. Análise de variabilidade, krigagens e funções de recuperação. In: SEMINÁRIO NACIONAL SOBRE INFORMÁTICA EM MINERAÇÃO: PESQUISA, LAVRA E BENEFICIAMENTO MINERAL, 6. Belo Horizonte - MG, IBRAM, p.60-69. 1996.

WACKERNAGEL, H. Multivariate Geostatistics: an introduction with applications, Berlim, Heidelberg: Springer-Verlag, 1995. 275 p.

\section{Artigo recebido em 11/05/2004 e} aprovado em 26/11/2004. 